livraisons

d'Histoire

de l'Architecture

\section{Livraisons de l'histoire de l'architecture}

35 | 2018

Femmes, architecture et paysage

\title{
Trois femmes paysagistes pionnières en France
}

Three women in Post-War French Landscape Architecture

Drei Landschaftsarchitektinnen als Wegbereiterinnen ihres Fachs in Frankreich

\section{Bernadette Blanchon}

\section{OpenEdition}

\section{Journals}

Édition électronique

URL : http://journals.openedition.org/lha/983

DOI : $10.4000 /$ lha. 983

ISSN : 1960-5994

Éditeur

Association Livraisons d'histoire de l'architecture - LHA

Édition imprimée

Date de publication : 15 juin 2018

Pagination : 125-138

ISSN : $1627-4970$

Référence électronique

Bernadette Blanchon, « Trois femmes paysagistes pionnières en France », Livraisons de l'histoire de l'architecture [En ligne], 35 | 2018, mis en ligne le 15 juin 2020, consulté le 25 janvier 2021. URL : http:// journals.openedition.org/lha/983; DOI : https://doi.org/10.4000//ha.983 


\section{Par Bernadette BLANCHON}

\section{TROIS FEMMES PAYSAGISTES PIONNIẼRES EN FRANCE}

"Ces années-là, sont une période difficile qui a forgé le caractère des gens et a développé leur capacité d'invention ».

Ingrid Bourne

Malgré leur contribution avérée à la construction de la figure contemporaine du paysagiste, les femmes ne sont que rarement mentionnées dans l'histoire de la discipline et de son enseignement ${ }^{1}$. Seule la sociologie des professions ${ }^{2}$ mentionne leur présence accrue dans la formation et la pratique. Ce champ de recherche, balbutiant en France, s'est développé plus précocement aux États-Unis ${ }^{3}$ ou en Europe du nord ${ }^{4}$, là où la culture et la société ont favorisé plus tôt à la fois le paysagisme et la place des femmes. Il nous a néanmoins été permis de participer à l'exploration de ce champ qui reste largement à développer, en revenant sur les figures féminines rencontrées lors nos travaux sur le paysage dans les ensembles de logements réalisés durant la période des Trente Glorieuses et la profession moderne de paysagiste en France ${ }^{5}$.

Aujourd'hui, les femmes représentent plus de $60 \%$ des étudiants qui intègrent le cursus de formation des architectes paysagistes en France ${ }^{6}$ et occupent la moitié des 130 postes d'architectes paysagistes-conseils de l'État, témoignant du chemin parcouru depuis la création, en 1945, du premier cursus de formation de paysagistes à l'école d'Horticulture de Versailles sise au Potager du Roi, la section du Paysage

1. Dorothée Imbert, "On site and time, French Landscape architecture in the twentieth century", Het landschap/ The Landscape, Antwerp, De Singel, 1995, p. 53-75 et Pierre Donadieu et Moez Bouraoui, "La formation des cadres paysagistes en France par le ministère de l'Agriculture 18742000 ", Rapport de recherche, ministère de l'Écologie et du développement durable, Cemagref, Bordeaux, 2003.

2. Chantal Cicé et Françoise Dubost, "La profession de paysagiste ", rapport de recherche, centre de sociologie des arts, EHESS, Mission de la recherche urbaine, 1986, p. 79-83.

3. Thaisa Way, Unbounded practice, Women and landscape architecture, in the early twentieth century, Charlottesville and London, University of Virginia Press, 2009; Duempelmann Sonja and Beardsley John (dir.), Women, Modernity, and landscape architecture, London \& New-York, Routledge, 2015.

4. "Gender, gardens \& garden history ", Bulletin för trädgardshitorik forskning, Stockholm, n 29, 2016.

5. Bernadette Blanchon, "Isabelle Auricoste (1941)"; Bernadette Blanchon \& Sonia Keravel, "France : paysagistes actuelles (nées après 1945) " in Didier et al. (dir.), Le Dictionnaire universel des créatrices, Paris, Éditions des femmes, Belin, 2013.

6. Statistiques du concours d'entrée commun aux formations de paysagiste DPLG des écoles de Versailles, Lille et Bordeaux ; années 2006 à 2012 (secrétariat du concours, École nationale supérieure du paysage de Versailles). 
et de l'Art des jardins. Il faut attendre la sixième promotion (1951-1953) de cette section du Paysage ${ }^{7}$ pour lire un nom féminin dans l'annuaire des anciens élèves. À partir des années 1970, elles sont plus nombreuses et atteignent la moitié d'une promotion qui compte une vingtaine d'étudiants ${ }^{8}$; malgré tout, peu d'entre elles mènent une pratique de conception. Dans l'école, elles restent souvent associées à une activité de jardinière ou plaisantées comme " cuscutes 9 ", présumées à la recherche d'une occupation et d'un mari.

L'enjeu majeur du cursus créé en 1945 était la dissociation du métier de paysagiste de l'exercice en entreprise, et la reconnaissance d'une "profession savante". Dès 1940, la création de l'Ordre des architectes avait interdit à ces professionnels le port de leur titre favori d' "architecte-paysagiste", les obligeant à se démarquer des architectes en démontrant la maîtrise de compétences spécifiques, à travers la création d'un enseignement et d'un diplôme. La section du Paysage, entendait alors offrir, selon les termes du décret de sa création, "la double série de connaissances requises, celle de l'horticulture et celle de l'architecture" pour former des professionnels destinés à intégrer les équipes chargées de la Reconstruction de la France. Cette période, dite des Trente Glorieuses (1945-1975), est celle de la construction des grands ensembles qui ont constitué un vaste champ d'expérimentation pour la profession de paysagiste en pleine redéfinition.

Les femmes, dont nous avons choisi de suivre les pas, sont à la fois des personnalités singulières et les témoins d'étapes significatives d'une évolution générale : la première, Ingrid Bourne, née en 1933, a joué un rôle essentiel pour ouvrir un milieu plutôt conservateur aux apports d'autres cultures. La seconde, Isabelle Auricoste, née en 1941, est la seule femme à avoir reçu le Grand Prix du Paysage ; elle offre l'exemple atypique d'engagements politiques et disciplinaires forts, nourris de passions littéraires. Enfin, Marguerite Mercier, née en 1946, très tôt impliquée dans la mâ̂trise d'ouvrage et l'approche d'échelles territoriales, a eu une pratique qui témoigne d'une diversification du métier confirmée aujourd'hui.

Ces trois personnalités ont vécu cette histoire récente avec la liberté et les contraintes propres aux femmes de leur époque. Nous les suivons ici dans leur enfance, dans leur formation à l'école de Versailles, longtemps seul cursus existant qu'elles ont traversé durant des périodes charnières, dans leur pratique professionnelle et à travers les avancées qu'elles ont pu susciter ${ }^{10}$.

7. Nous nommerons ici désormais "section du Paysage " la section du Paysage et de l'Art des jardins. Cette formation est à l'origine de la création en 1976 de l'École nationale supérieure du paysage, établissement reconnu aujourd'hui dans le monde.

8. "Section du Paysage 1946-1972 ", Annuaire des Anciens Élèves de l'ENSH et de l'ENSP, 1994, Versailles, Association des ingénieurs horticoles et anciens élèves de l'École nationale supérieure d'horticulture et de l'école nationale supérieure du paysage, 1994, p. 96.

9. Parasite de la luzerne et sobriquet dont les élèves ingénieurs de l'École d'horticulture gratifient les apprentis paysagistes. Il est repris par ces derniers à l'attention des auditeurs libres et des demoiselles.

10. Pour un document plus complet, voir Bernadette Blanchon, "Creative margins: Three women in post-war French landscape architecture ", dans Duempelmann S. and Beardsley J. (dir.), op. cit., p. $103-121$. 


\section{Ingrid Bourne, des références nouvelles venues du nord de l'Europe}

Ingrid Bourne, née Cloppenburg, grandit en Allemagne durant la Seconde Guerre mondiale, dans une famille d'origine hollandaise et apprend tôt l'art de tourner toute contrainte en opportunité. Passionnée de plantes, elle étudie dans une école d'agriculture en Angleterre avant d'intégrer, en auditrice libre, la section du Paysage à Versailles en 1954. Elle restera marquée par les paysages ouverts de la mer Baltique, tenus sous le ciel par des digues immenses ainsi que par les grandes étendues de pelouses ponctuées d'arbres fruitiers de la campagne anglaise. Mais Versailles est une révélation : la ville et son plan, avant même les jardins, la fascinent. Sa culture différente est un ferment stimulant dans les échanges avec ses camarades et professeurs, comme Théodore Leveau (1896-1971), un architecte urbaniste inspiré par le «Grand siècle Français ». Elle rapporte notamment des expériences issues de ses stages d'été en Allemagne : les associations végétales, constituées d'essences champêtres, ou "indigènes ", apprises auprès de Karl Plömin auteur du très populaire parc d'exposition Planten un Blumen ${ }^{11}$ à Hambourg (ill. 1) ou les autoroutes, conçues par Alwin Seifert (1890-1972) sous le III ${ }^{\mathrm{e}}$ Reich, afin de « recoudre le paysage lors du passage de l'autoroute ». Plus qu'un matériau, la plante est considérée comme un être vivant à respecter, le site comme la matière du projet, quand en France les talus de routes et les parcs sont tapissés de rosiers et de plantes ornementales vives. "Chaque paysage a une histoire qu'il ne faut pas nier, [...pas plus que] la couleur, les tissus et le rythme qui le caractérisent ${ }^{12}$. " Forte de ces références, auxquelles il convient d'ajouter les travaux du Suisse Willi Neukom (1917-1983), elle développera ce credo paysagiste avec son époux Michel Bourne, dans leur association professionnelle près de Lyon. À l'instar de ces figures fondatrices, ils réalisent ensemble des séries de relevés de situations existantes afin d'en comprendre les rythmes de plantations, les associations d'essences et d'espèces ... et d'en transposer le potentiel dans leurs nouveaux projets. Michel Bourne dirige l'entreprise de jardins et suit les chantiers, tandis qu'Ingrid se consacre à la conduite du bureau d'étude, en même temps que naissent quatre enfants.

\section{Premier défi : pragmatisme et invention}

Pour faire face à une première commande d'un nouveau type, l'aménagement paysager de l'Unité de voisinage de Bron-Parilly ${ }^{13}$, Ingrid s'installe dans les bureaux des architectes de l'opération, alors que Michel est appelé sous les drapeaux. Cet ensemble expérimental de logements sociaux est réalisé dans l'enthousiasme de la Reconstruction, sans que personne ne se soucie de l'espace entre les bâtiments, ici séparés des voiries par des dénivelées de plusieurs mètres! En l'absence de budget et

11. Voir : Heino Grunert, «75 Jahre Planten un Blomen, Hamburgs Niederdeutsche Gartenschau von 1935 ", Stadt+Grün, novembre 2010, p. 51-59.

12. Alwin Seifert cité par M. et I. Bourne, entretien avec l'auteur, Lyon, 23 octobre 2013.

13. Unité de voisinage de Bron-Parilly, "Secteur Industrialisé", OPHLM du Rhône, 1951-1963, architectes Pierre Bourdeix, René Gagès et Franck Grimal, 30 ha, 2607 logements. 


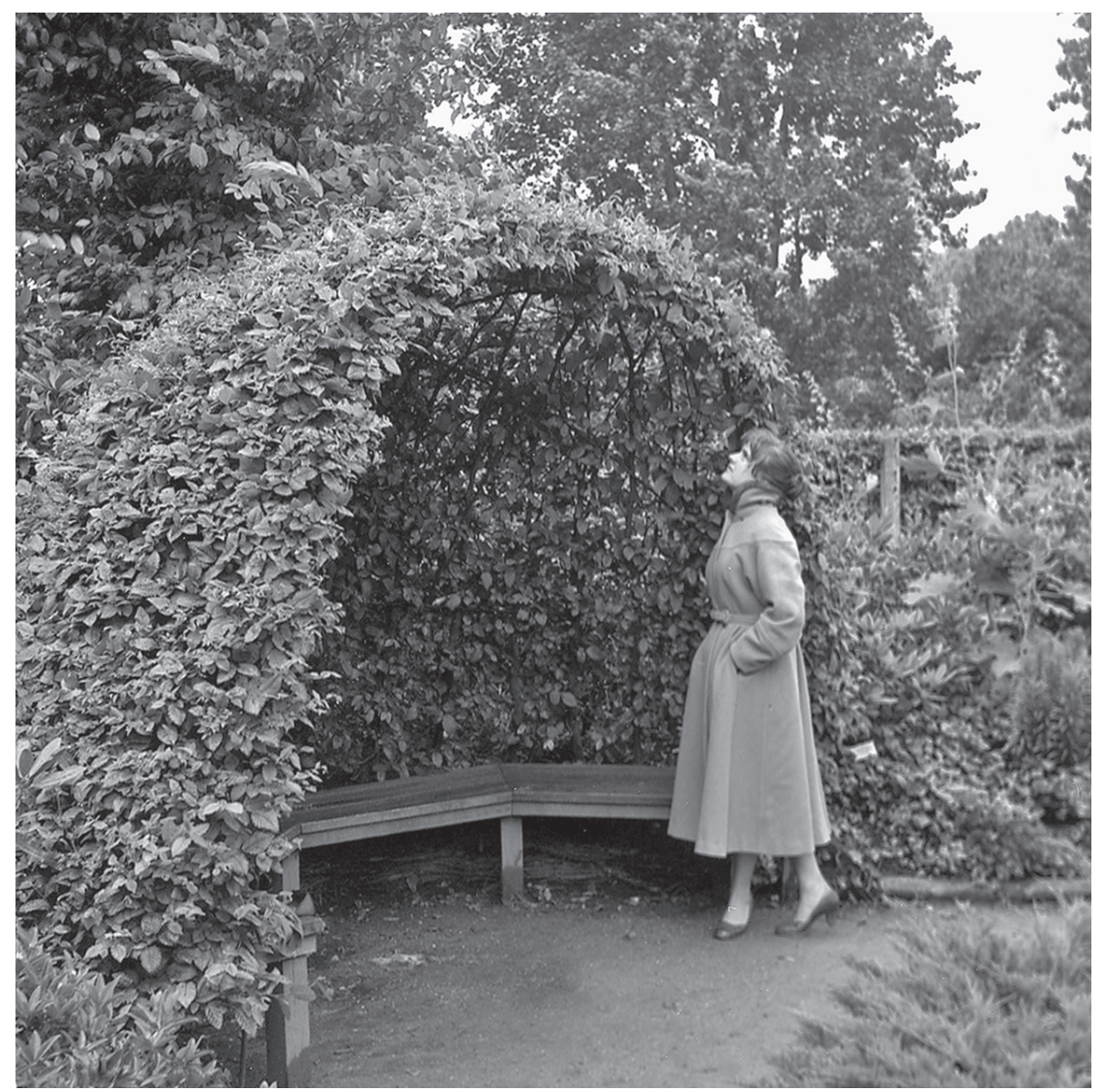

Ill. 1 : Ingrid Bourne visitant le jardin public Planten un Blomen, Hambourg, 1956. (C) Ingrid et Michel Bourne.

de relevés, le travail est mené in situ, à l'aide d'engins de travaux publics et agricoles pour modeler le sol et semer les hectares de pelouse. "On n'avait jamais fait ça. Il fallait inventer et exécuter au fur et à mesure ${ }^{14}$ ». Les plantations d'essences locales, rustiques, platanes, peupliers, tilleuls, quelques pins - adaptées aux sols tassés stériles, sont réalisées selon des techniques de plantations forestières, comme en Allemagne. "Il fallait le courage d'attendre 4-5 ans que cela devienne joli ! ${ }^{15}$ " Les alignements végétaux, en pied d'immeubles, évoquent les lignes de vergers voisins et les masses végétales en bosquets "comme dans les dessins de Le Corbusier ${ }^{16}$ ", protègent les habitants des nuisances des infrastructures routières, tout en constituant des espaces différenciés (ill. 2).

14. Ingrid Bourne, entretien avec l'auteur, Strasbourg, 15 octobre 2013.

15. Idem.

16. Michel Bourne, entretien avec l'auteur, Lyon, 20 novembre 1995. 


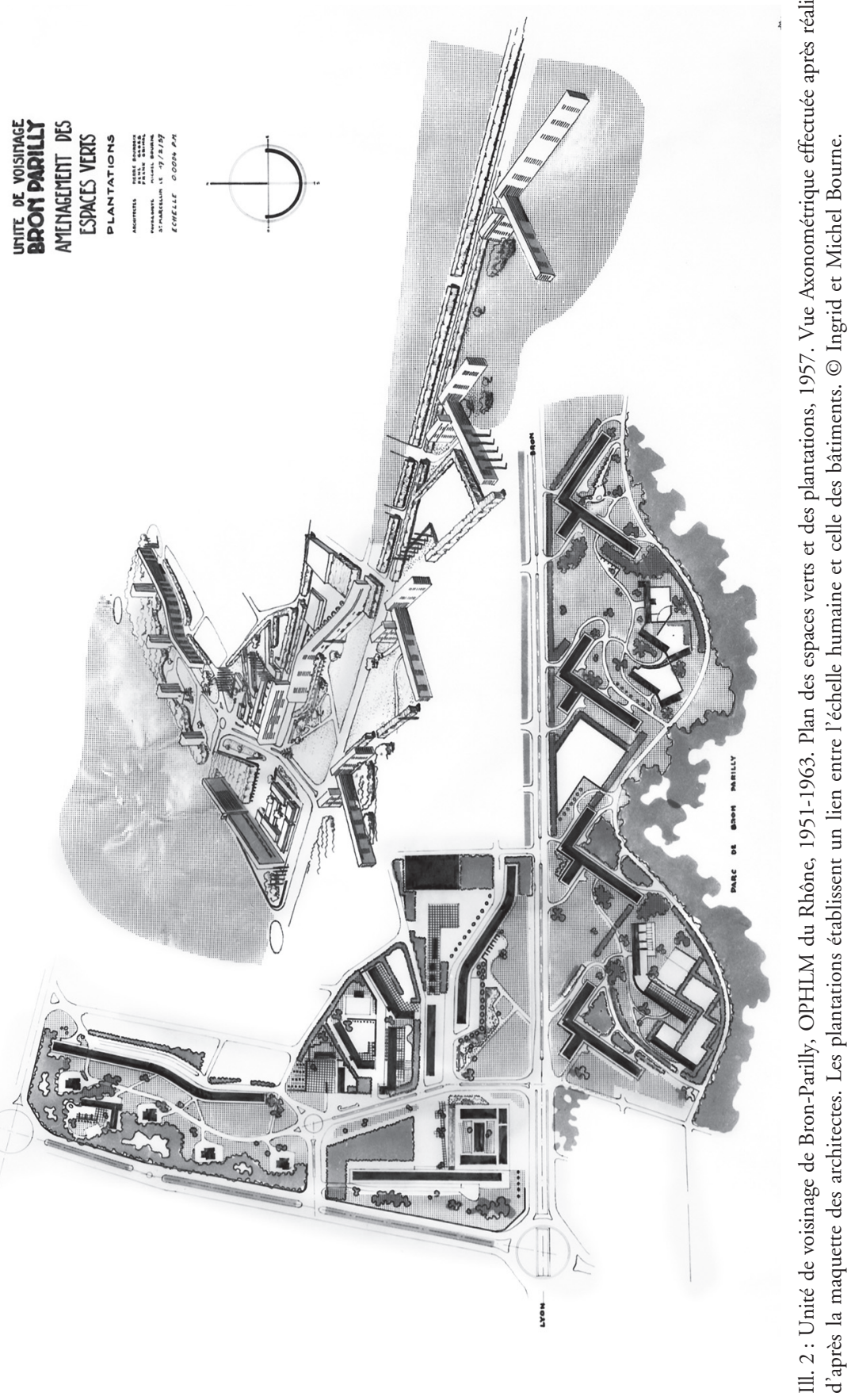

+0 


\section{Reconnaissances}

Bientôt reconnus et associés à la plupart des chantiers de grands ensembles de la région, ils peuvent céder l'entreprise de jardins et espaces verts familiale qu'ils géraient parallèlement à leur bureau d'études pour se consacrer entièrement à la conception paysagère. À partir de 1967, ils ouvrent un Atelier de Paysage à Saint-Marcellin, élargissant leur domaine d'activité aux campus, villes nouvelles et espaces publics... Ingrid Bourne suit désormais ses propres projets, de la conception à la réalisation, développant une écriture personnelle plus libre, à la recherche d'un équilibre entre structure et souplesse du végétal, entre géométrie et aléatoire du milieu vivant, " entre Le Nôtre et Plömin ${ }^{17}$ ". Deux projets notamment en témoignent : celui du Parc Paul Mistral (1966-1971) à Grenoble, ouvert sur la ville pour accueillir les jeux Olympiques d'hiver de 1968 , et celui de la ville neuve d'Échirolles ${ }^{18}$, à l'ouest de Grenoble, pour lequel elle participe à la programmation, la concertation et la mise en ouvre de nouveaux quartiers autour d'un parc central de 9 ha. Chaque projet est l'occasion de mobiliser sa culture internationale, d'affirmer son goût du végétal et de l'écoute du client. Elle propose une formule très appréciée d'accompagnement des particuliers dans le dessin de leur projet de jardin, suivant leurs goûts, leur budget et le temps qu'ils consacrent à leur entretien. Dans les années 1990, les Bourne accèdent à des commandes prestigieuses à Lyon, de l'aménagement de la Place Antonin Poncet jusqu'aux jardins créés par Ingrid sur la colline de Fourvières (2000). Ils installent ensuite, pendant quelques années, leur bureau en Allemagne où ils sont aujourd'hui retraités.

\section{Les années 1950-1960: un nouveau métier dans une société en mutation}

Son éducation a développé chez Ingrid Bourne un sens précoce des responsabilités, qui lui a permis de s'imposer dans bien des situations où certains acceptaient mal d'être dirigés par une femme, jeune qui plus est. "Quand on est sûre de son projet, qu'on l'a dessiné soi-même et quand on l'a dans la peau, personne ne peut vous attaquer ${ }^{19}$." La pratique professionnelle du couple est caractéristique du passage de l'entrepreneur au concepteur paysagiste, à travers une histoire liée à la reprise d'une entreprise familiale en région. D'autres, plus parisiens, ont pu suivre des compléments de formation et faire évoluer l'enseignement de la section du Paysage, comme Jacques Sgard ${ }^{20}$, développant par d'autres voies des références issues des cultures d'Europe du nord et de l'est qui avaient déjà été mises en avant par Ingrid Bourne. On relève notamment la création d'un cours d'écologie et de phytogéographie par Jacques Montaigut (1925-2007) à partir de $1967^{21}$. C'est à ce moment charnière, de renouvellement de la pédagogie qu'Isabelle Auricoste rejoint la section du Paysage.

17. Ingrid Bourne, entretien cité, 2013.

18. 5000 logements de 1967 à 1985.

19. Ingrid Bourne, entretien cité, 2013.

20. Voir Blanchon Bernadette, «Les paysagistes en France depuis 1945 : l'amorce d'une indiscipline ou la naissance d'une profession ", in Les espaces publics modernes, Virginie Picon-Lefebvre (dir.), Paris, Le Moniteur, 1997, p. 121-210.

21. Conversation avec Pierre Donadieu, janvier 2014. Voir Marc Rumelhart, "Éco-logiques pour les projets de Paysage, autobiographie d'un héritage ", Carnets du Paysage, no 20, novembre 2010, p. 196-197. 


\section{Isabelle Auricoste : le paysage, levier de transformations sociétales}

Pour Isabelle Auricoste, cette époque d'expansion qui suit la guerre est à la fois celle de la libération et celle de l'aliénation par la consommation et le confort moderne. Née en 1941, elle passe son enfance en Beauce, entre des parents artistes, et se dit fille de la campagne. «(Ce) milieu d'origine artistique, m'a donné un bain de culture et des clefs de lecture implicites qu'il me semble avoir mobilisés toute ma vie ${ }^{22}$." D'abord inscrite à l'institut d'Urbanisme de l'université de Paris, elle se passionne ensuite pour l'histoire de l'art, la sociologie et l'anthropologie à l'École Pratique des Hautes Études $(\mathrm{EPHE})^{23}$. Ces enseignements permettront ces allers-retours entre engagements politiques et réflexions humanistes qui guideront sa pratique. Évoquant les cours du sociologue Henri Lefebvre, elle rappelle qu'il était "convaincu que l'urbanisme et l'architecture avaient le pouvoir de changer la vie ${ }^{24}$ ». Elle intègre la section du Paysage en 1961 pour se confronter à une activité concrète et sacrifier à ses aspirations de vie au grand air, et bénéficie du renouvellement des programmes offerts par de jeunes professionnels, impliqués notamment dans les aménagements des nouvelles "zone à urbaniser en priorité " (ZUP). Elle se tient cependant à l'écart d'une institution conservatrice qui lui interdit de passer le diplôme pour avoir participé à une manifestation en faveur du Front de Libération Nationale algérien (FLN) et ne cherchera jamais à acquérir le titre de "diplômé par le gouvernement " (DPLG). Restée proche des milieux de l'architecture et de l'EPHE, elle participe, avec Hubert Tonka son époux, à la fondation d'un groupe marxiste de mouvance " conseilliste " et à celle de la revue Utopie, publiée de 1967 à 1978. Son engagement se poursuit dans une vie en communauté autour d'une imprimerie en coopérative à Fontenay-sous-Bois où elle installe son agence de paysagiste en 1966, choisissant ce qu'elle qualifie comme « une profession nouvelle, en train de s'inventer, [qui donnait] moins l'impression d'être dans le système ${ }^{25}$ ». Enceinte pendant son premier chantier, elle accouche d'une petite fille née alors que paraît le premier numéro d'Utopie, en 1967. Tout est mené de front, dit-elle, sans "problèmes particuliers en tant que femme 26 ".

\section{Les espaces verts : du blanc du papier aux espaces de vie quotidienne}

L'aménagement des quartiers de logement sociaux dans les banlieues parisiennes constitue l'essentiel de son travail durant douze années, à Sartrouville ${ }^{27}$ et à Bobigny ${ }^{28}$

22. Isabelle Auricoste, entretien avec l'auteur, Poitiers, 25 octobre 2013.

23. La VI ${ }^{\mathrm{e}}$ section de l'EPHE, Sciences économiques et sociales, dont l'autonomisation en 1975 donnera lieu à la création de l'EHESS, École des hautes études en sciences sociales.

24. Isabelle Auricoste, entretien cité, 2013.

25. Isabelle Auricoste, entretien cité, 2013.

26. Isabelle Auricoste, entretien avec l'auteur, Paris, 9 mars 2010.

27. Cité des Indes, ZUP de Sartrouville, Roland Dubrulle architecte, 3000 logements, 1967-1981.

28. Cités Karl Marx et Paul Éluard, ZAC (Zone d'aménagement Concerté) de Bobigny, Michel Holley architecte, 1971-79. 


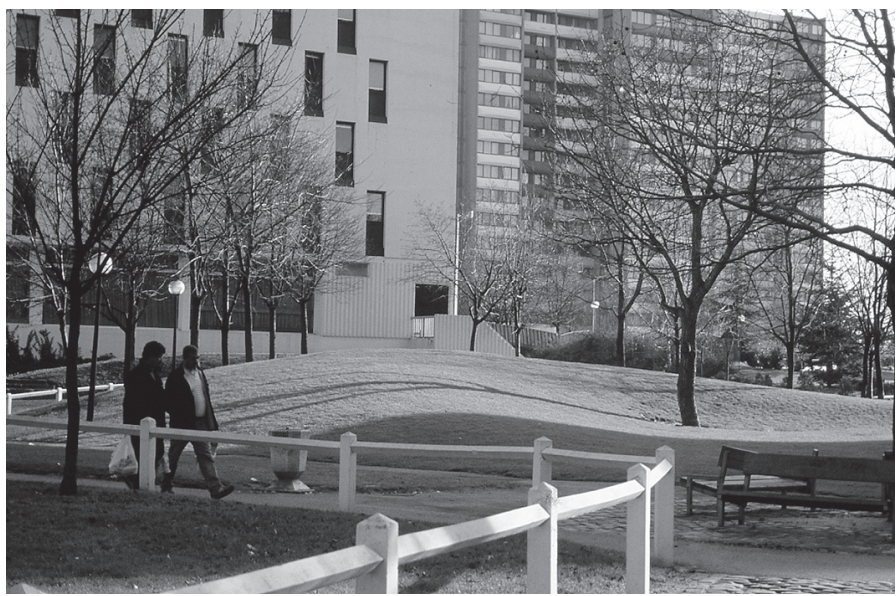

Ill. 3 : Bobigny, centre ville, 1975. Modelé de sol « apprivoisant » l'espace entre les routes et les bâtiments. Photo 1988. (C) Isabelle Auricoste.

(ill. 3). Là encore, le grand ensemble est la solution consensuelle à la crise du logement, mais personne ne maîtrise ce «blanc du papier entre bâtiments et circulations. [...] Nous n'avions pas appris, mais nous le faisions! Du gazon, des arbres... ${ }^{29}{ }^{\prime}$. Pour apprivoiser l'étendue entre les bâtiments, les modelés de sol reprennent le maniement des courbes de niveau, enseigné par Sgard, et l'usage « d'essences locales, le moins exotiques possible par refus de la tradition jardinière-horticole ${ }^{30}$ ", cette tradition faisait en effet appel, depuis le XIX ${ }^{e}$ siècle, à des effets pittoresques s'appuyant notamment sur l'introduction de plantes exotiques et l'hybridation. Vers 1980, elle cesse son activité pour privilégier l'écriture et sacrifier à son goût pour la littérature anglaise, avant de s'impliquer peu à peu dans la pédagogie. Elle enseigne au sein de ce qui était devenu l'école nationale supérieure de Paysage de Versailles (1981-1990), puis participe à la création d'un enseignement d'histoire de l'art des jardins à l'école d'architecture de Versailles, qui donnera naissance à une formation sur le patrimoine des jardins historiques ${ }^{31}$; en 1991, elle rejoint l'équipe fondatrice de l'école de Paysage de Bordeaux (ill. 4).

\section{Le Paysage dans les dynamiques territoriales}

Installée depuis lors en Charente où elle a fondé l'atelier Mandragore, elle restaure des jardins historiques et réalise des projets d'espaces publics, parfois en association avec de jeunes professionnels, comme la place de la gare TGV à Tours (1991-92)

29. Isabelle Auricoste, entretien cité, 2010.

30. Isabelle Auricoste, entretien cité, 2013.

31. L'actuel Master Jardins Historiques, Paysages et Territoires, initié en 1992 avec Janine Christiany et Monique Mosser, historienne de l'art des jardins. 


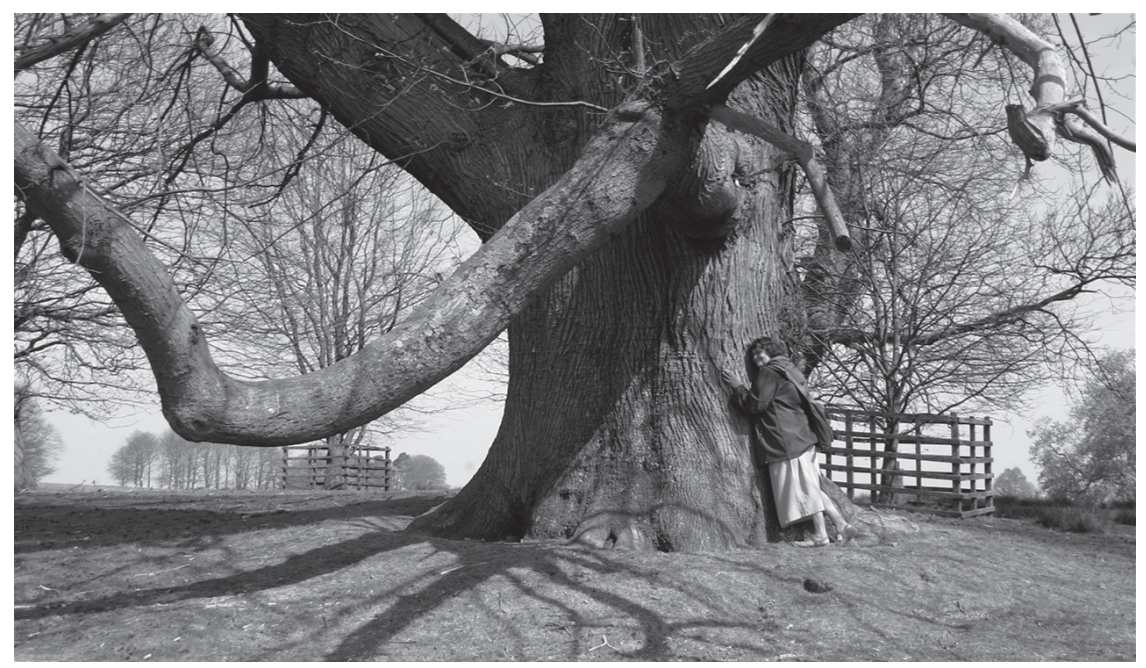

Ill. 4 : "Fagus Hug ". Isabelle Auricoste enserrant un hêtre, voyage d'étude en Angleterre, Petworth, 2007. (C) Serge Briffaud.

avec le paysagiste Yves Brunier ${ }^{32}$ (1962-1991). Elle a reçu, en 2000, le premier et seul Grand Prix du paysage attribué à une femme. Aujourd'hui retraitée de l'enseignement, maire-adjointe de sa commune, présidente du schéma de cohérence et d'organisation territoriale (SCOT) et vice-présidente du syndicat du Pays Ruffecquois (90 communes), elle poursuit une activité engagée dans la gestion du paysage rural, à la recherche de leviers de transformation permettant à la maîtrise d'ouvrage publique d'agir sur des espaces menacés par les intérêts privés ou les logiques sectorielles.

\section{Marguerite Mercier, au service des politiques publiques de paysage}

Cette implication en amont des projets est aussi caractéristique du parcours de Marguerite Mercier dont la personnalité témoigne de l'engagement dans un métier qui " a structuré [son] existence et [1]'a construite en tant qu'être humain "; plus qu'une expérience professionnelle, "l'aventure d'une vie ${ }^{33}$ ". Née en 1946, elle suit la formation de la section du Paysage, de 1965 à 1967, et bénéficie des nouveaux enseignements déjà appréciés par Isabelle Auricoste, dont l'écologie avec Jacques Montaigut et "La perception vivante des lieux " avec Bernard Lassus. Elle approfondit sa formation à l'institut d'Urbanisme de l'université de Paris où elle aborde

32. Collaborateur de Rem Koolhas et de Jean Nouvel. Voir Yves Brunier, Landscape Architect, paysagiste, Bordeaux, Arc en rêve/Birkhauser, 2001.

33. Marguerite Mercier, "Une aventure de vie », Blanquefort, septembre 2007, manuscrit non publié. 
l'histoire du développement urbain et suit l'embrasement des sociologues en 1968, autour d'Henri Lefebvre, "pour une nouvelle société utopique, libre et généreuse ! ". À partir du socle de ces deux formations, sa pratique l'a constamment fait évoluer.

\section{Vers la commande publique et l'enchaînement des échelles de l'aménagement}

Après une brève expérience chez des architectes, sur un projet de grand ensemble, elle s'installe en libéral et engage d'emblée une prospection militante auprès de commanditaires potentiels (structures territoriales de l'Agriculture ou de l'Équipement, Parcs Naturels, sociétés d'aménagement, office national des Forêts ...). Pour vaincre sa timidité, elle accompagne cette démarche d'argumentaires élaborés montrant les enjeux publics de l'approche paysagiste. Elle se nourrit des lectures des revues Anthos ou Urbanisme, également citées par ses consœurs. Peu à peu, elle intervient comme salariée au sein de structures institutionnelles où elle s'implique pour la cohérence des projets, de la conception à la réalisation. De 1972 à 1976, elle travaille à l'établissement public de la Ville nouvelle de Saint-Quentin-en-Yvelines sur l'armature d'espaces publics de la ville, puis, de 1976 à 1989, au sein de la Mission Interministérielle pour l'aménagement de la Côte Aquitaine (MIACA), où la conduit le besoin de se rapprocher d'un milieu naturel, ouvert sur le ciel et l'océan. Cette mission met alors en place un des grands projets d'aménagement touristique de l'État initié par la Délégation interministérielle à l'aménagement du territoire et à l'attractivité régionale (DATAR). Durant douze années de travail expérimental et intense, Marguerite Mercier démontre comment une approche environnementale peut se décliner dans ses dimensions paysagère, écologique et forestière. Elle est là, un relais entre la commande publique et sa mise œuvre, et cela, d'autant plus qu'elle est elle-même conceptrice. Elle élabore les programmes d'intervention paysagère et les cahiers des charges afin que des professionnels libéraux puissent œuvrer facilement à l'aménagement de plages, de campings et de stations touristiques en forêt (ill. 5). Elle vérifie la cohérence de l'espace construit à travers les différentes échelles d'aménagement et la continuité de mise en ouvre entre espaces publics et privés, inventant les conditions d'un travail pluridisciplinaire entre experts, concepteurs et aménageurs, tout en maintenant un équilibre entre les apports de sa connaissance, son savoir-faire professionnel et le respect du rôle du maître d'œuvre chargé du projet. Avec patience et effacement, elle cherche les leviers d'intervention et attend les moments propices à leur concrétisation.

\section{L'aventure du service public}

Suivront dix-sept années d'implication à la direction départementale de l'Équipement (DDE) de la Gironde durant lesquelles elle a partagé la culture du projet de paysage avec ses collègues, des partenaires externes, ainsi que d'autres acteurs des services de l'État et des collectivités locales. Enfin, sollicitée pour enseigner à l'école d'architecture de Bordeaux, elle mesure les limites de l'exercice et, avec Caroline Stefulesco ${ }^{34}$ alors présidente de la Fédération française du paysage, elle émet l'idée 


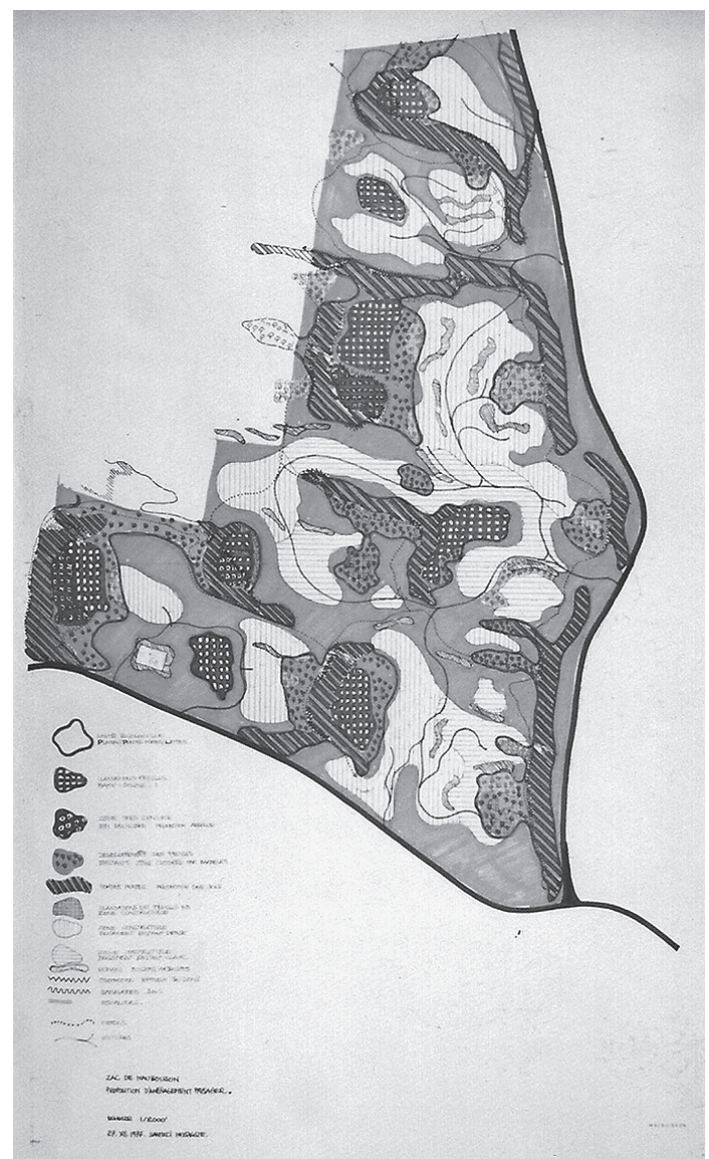

Ill. 5 : Plan couleur de l'aménagement concerté du site de Carcans Maubuisson, côte aquitaine, 1979. (C) Marguerite Mercier. Paysage et aménagement de nouveaux villages de vacances. Extrait de : Marguerite Mercier, Aménagement et Paysage, 11 réalisations, MIACA, décembre 1988.

de créer une nouvelle école pour former davantage de paysagistes. Mais lorsque le projet voit le jour, en 1991, sa charge de travail à la DDE de Bordeaux ne lui permet pas d'y participer. Marguerite Mercier est consciente d'avoir eu la chance de participer à des aventures d'une ampleur exceptionnelle, impossibles aujourd'hui dans le contexte d'une décentralisation croissante ${ }^{35}$. Cette pratique au service de l'intérêt public est une leçon d'humilité qui lui a "appris à s'incliner devant la nature, sa force et sa fragilité ; [...] l'importance de laisser la place au temps pour que les milieux naturels se reconstituent [...] ; la priorité à donner à la gestion qu'elle soit du milieu naturel ou des espaces plantés ${ }^{36}$ " (ill. 6). Elle a eu un impact sur de nombreuses

35. Marguerite Mercier, "Une aventure de vie", op. cit., p. 6.

36. Marguerite Mercier, entretien téléphonique avec l'auteur, 4 janvier 2014. 


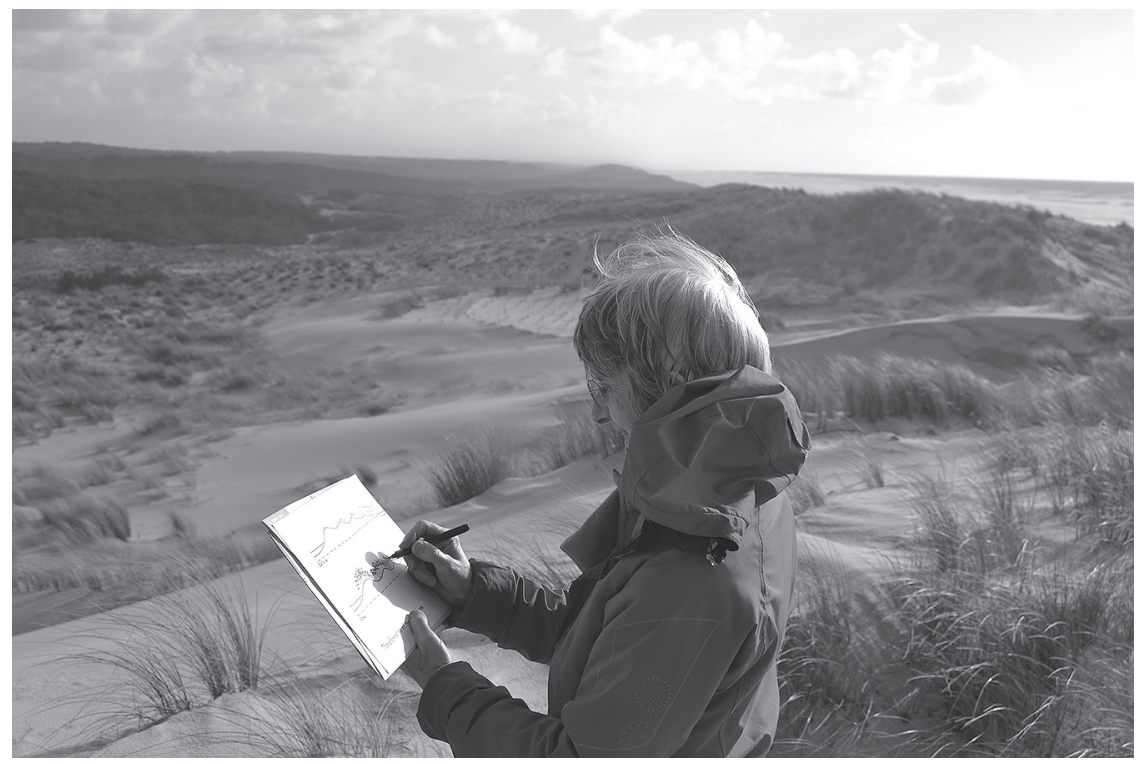

Ill. 6: Marguerite Mercier sur la dune entre océan et forêt brossant le 16 février 2014, un schéma explicatif de protection du littoral, après les forts orages du début de l'année, à l'attention des habitants. Les dunes à l'arrière-plan ont été stabilisées par des plantations au XIXe siècle. () Marguerite Mercier.

actions régionales, mais reste mal capitalisée et souvent ${ }^{37}$ ignorée des équipes en charge de ces sites aujourd'hui. Marguerite Mercier se décrit elle-même comme " une plante pionnière, celle qui traverse le feu et prépare le sol pour que les espèces suivantes s'installent ${ }^{38}$ ".

\section{Trois femmes engagées}

Ces trois créatrices ont suivi chacune un itinéraire original que nulle femme n'avait connu avant elles en France. La liberté avec laquelle elles ont su contribuer au renouvellement des pratiques paysagistes, témoigne de leur détermination autant que d'une souplesse et d'une ouverture toujours fécondes dans leurs modes d'exercice et dans la transmission des idées. Ingrid Bourne peut être considérée comme une pionnière européenne d'une pensée écologique assez bien partagée aujourd'hui ; Isabelle Auricoste comme une militante d'une pensée socio-politique du paysage indissociable d'un fort investissement pédagogique et de l'inscription dans une culture artistique et humaniste; Marguerite Mercier comme une pionnière du service des

37. Conférence, "La Miaca qu'est-ce? L'aménagement de la côte Aquitaine, quels résultats ? ", Cycle Expériences de Paysage, Fédération Française du Paysage (FFP), Pavillon de l'Arsenal 30 avril 2011, http://www.f-f-p.org/fr/experiences-de-paysage/?id=4\&max=8\&st=16 (consulté 2013/12/23).

38. Marguerite Mercier, "Une aventure de vie", op. cit., p. 8. 
pouvoirs publics, très tôt impliquée dans la maîtrise d'ouvrage en amont des opérations et toujours en lien avec une pensée concrète de l'espace.

On retrouve ces approches dans la pratique de figures masculines plus connues : internationale, jardinière et écologique, chez un Gilles Clément ; pédagogique et politique chez Michel Corajoud ou chez Alexandre Chemetoff qui entend former les commanditaires; plus institutionnelle chez Pierre Dauvergne ${ }^{39}$, qui a œuvré dans le même sens que M. Mercier.

Le souci de la cohérence entre les échelles d'intervention, depuis l'amont et l'énoncé de la commande, est récurrent. Toutes trois ont tissé leurs apports respectifs de manière particulière dans leur pratique et permis à chaque fois la redéfinition d'une commande d'abord limitée et floue, au travers de ce qu'on pourrait appeler "l'invention programmatique ", comme partie prenante du projet de paysage. Elles ont notamment cherché à mieux définir la commande publique et à en rendre les ressorts transmissibles au niveau institutionnel et pédagogique. Avec leurs consœurs et confrères pionniers et militants, elles ont ainsi contribué indirectement à l'évolution des institutions et des politiques publiques, en donnant une place centrale à leur adaptation au caractère évolutif du paysage. Malgré tout, elles apparaissent peu dans l'histoire d'une période qui déjà laisse peu de place au paysage et aux paysagistes. Les raisons de cet oubli résident aussi dans leurs situations particulières : Ingrid Bourne comme épouse d'un associé plus expansif ; Isabelle Auricoste souvent éloignée de la nébuleuse versaillaise et concentrée sur des problématiques plus rurales qu'urbaines. Marguerite Mercier, enfin, moins visible et moins susceptible d'apparaitre au premier plan dans la maitrise d'ouvrage et les institutions publiques. Elles partagent un gout limité pour la médiatisation et ses images réductrices ainsi que la conviction que la pratique paysagiste peut contribuer à améliorer l'avenir de la société.

Inspirées par les espaces ouverts qui les émeuvent et les ressourcent : la Baltique, la campagne anglaise, la côte Aquitaine, la Charente, elles ont engagé leur vie au-delà de leur métier, comme, dit Marguerite Mercier, " un privilège pour acquérir une vision exceptionnelle du monde, à la fois de plus en plus large et de plus en plus attentive aux détails, [...] pour apporter une qualité d'observation et de lecture des problématiques très particulière ${ }^{40}$. " Cette généalogie féminine ouvre, aux marges des modèles français dominants, un champ fécond que d'autres figures se prêteraient à alimenter. Un champ particulièrement favorable aux femmes? Celles-ci semblent, en effet, nombreuses à s'accorder avec les spécificités de l'approche paysagiste : qualités d'observation et de compréhension des situations, écoute des clients et usagers, soumission aux lois du milieu vivant.... et à opter pour une méthode systémique et

39. Né en 1943, il intègre la section du Paysage en 1962. Il a été directeur des espaces verts du Val-deMarne (1984), puis directeur de l'aménagement urbain (1991) et directeur des services techniques de l'aménagement, de l'environnement et de l'assainissement du Val-de-Marne. Voir Michel Racine (dir.), Créateurs de jardins et Paysages du XVIe au XXIe siècle, t. 2, 2002, ENSP-Actes Sud, p. 302-305.

40. Marguerite Mercier, entretien téléphonique avec l'auteur, 24 janvier 2014. 
empathique qui entre de plus en plus en résonnance avec les problématiques économiques, environnementales et sociétales actuelles, dont on peut tirer les fils précoces comme nous l'avons ébauché ici.

Bernadette BLANCHON architecte DPLG, maitre de conférences, ENSP de Versailles 